\title{
L'E-assurance : La Présentation à Distance des Opérations d'assurances au Maroc, Quel Apport du Cadre Juridique! (Période 2004 à fin 2019)
}

\author{
Hicham Rahal, Docteur Ingénieur \\ Enseignant Chercheur à l'Ecole Nationale de Commerce et de Gestion de \\ Kénitra, Université Ibn Tofail kenitra, Morocco
}

Doi:10.19044/esj.2020.v16n22p306 URL:http://dx.doi.org/10.19044/esj.2020.v16n22p306

\section{Résumé}

Cet article se propose de faire un état des lieux du cadre juridique de la vente à distance dematérialisée (VAD) de l'assurance au Maroc à travers l'analyse des dispositions du code des assurances promulgué en 2004, de l'étude de la circulaire ${ }^{\circ} \mathrm{DAPS} / \mathrm{EA} / 12 / 19 \mathrm{du} 09$ mars 2012 base réglementaire de la VAD dématérialisée de l'assurance et de présenter certaines données du secteur des assurances à fin 2018. De plus, l'étude du cadre juridique de présentation des opérations d'assurances à distance fait ressortir certaines faiblesses, c'est ce qui a été démontré à travers une analyse critique. La méthodologie adoptée consiste à répondre à certaines questions posées au départ, pour établir s'il y a un changement significatif au niveau du cadre juridique de présentation à distances des opérations d'assurances en premier lieu et de voir si ce cadre juridique réponds aux attentes des opérateurs et du consommateur en matière de vente à distance de manière dématérialisée de l'assurance (l'e-assurance). Il ressort de l'étude que le législateur marocain à introduit dans le circuit législatif un projet de loi modifiant le code des assurances en integrant un volet dédié à la présentation à distance des opérations d'assurances. Ce projet de loi reprend les dispositions de la circulaire de la vente à distance $n^{\circ} \mathrm{DAPS} / \mathrm{EA} / 12 / 19$ sans changement majeur. D'ailleurs, ce cadre juridique ne permet pas de dématérialiser totalement le processus de vente à distance puisque les opérateurs d'assurances dans la phase ultime du contrat d'assurances utilisent le contrat papier. La dématérialisation est imparfaite.

Mots clés : Vente à distance dématérialisée, l'e-assurance, cadre juridique de présentation de l'e-assurance, champ d'application, les limites de la présentation des opérations d'assurances 


\title{
E-insurance: The Remote Presentation of Insurance Operations in Morocco, What a Contribution of the Legal Framework! (Period from 2004 to the End of 2019)
}

\author{
Hicham Rahal, Docteur Ingénieur \\ Enseignant Chercheur à l'Ecole Nationale de Commerce et de Gestion de \\ Kénitra, Université Ibn Tofail kenitra, Morocco
}

\begin{abstract}
This paper aims to make an inventory of the legal framework of the dematerialized distance selling (DS) of insurance in Morocco. This is done through the analysis of the provisions of the insurance code promulgated in 2004 and the study of the circular $n^{\circ}$ DAPS/EA/12/19 of March 9, 2012, which is the regulatory basis of the dematerialized DS of insurance. The study also presented some data of the insurance sector at the end of 2018. The study of the legal framework for the DS of insurance operations also highlights some weaknesses. The methodology adopted provides answers to certain questions posed at the outset. This is aimed at establishing if there is a significant change in the legal framework for distance selling of insurance operations. This also helps to ascertain if this legal framework meets the expectations of operators and consumers for dematerialisation of distance selling of insurance operations (e-insurance). The study shows that the Moroccan legislator has introduced a bill to amend the Insurance Code by including a section dedicated to distance selling of insurance operations. This bill takes up the provisions of the circular of distance selling $n^{\circ}$ DAPS/EA/12/19 without any major change. This legal framework, however, does not provide a complete dematerialization of the distance selling process since insurance operators in the final phase of the insurance contract use the paper contract. The dematerialisation is also not fully applied.
\end{abstract}

Keywords: Dematerialized distance selling, e-insurance, a legal framework for the presentation of e-insurance, scope, weaknesses of the presentation of insurance operations

\section{Introduction}

Le commerce électronique à distance avec internet a révolutionné le monde des affaires. Et dans le but de prévoir un cadre juridique adapté aux 
services financiers, qui est une composante du monde des affaires, l'Organisation Mondiale du Commerce consciente de l'importance que revêtent les services financiers dans l'économie moderne, a engagé dès 1997, des négociations en vue d'assurer une parfaite libéralisation de ces services, afin d'abolir les distorsions qui existent entre les différents marchés financiers. Ainsi, dématérialisé, les services financiers ont pu librement circuler sur la toile, profitant des potentialités qu'offre un marché mondial de biens et de services.

On peut considérer qu'Internet constitue un réseau ouvert particulièrement adapté à la commercialisation des biens et services financiers, du fait qu'il en a facilité la gestion et la circulation, par la suppression, entre autres, des frais de transport et de logistique. Il faut souligner que le commerce électronique ne se serait certainement pas développé sans la dématérialisation des mécanismes financiers. Le développement du premier n'aurait pu se réaliser sans la dématérialisation des seconds. En effet, comment un commerçant aurait pu commercialiser son produit en ligne, proposer de bas prix, si le client internaute n'avait pas la possibilité de payer en ligne avec les moyens financiers électroniques adéquats (carte bleue, porte-monnaie électronique...) (Boulaich Bayssa, 2012).

Le Maroc ne fait pas exception, il s'inscrit dans cette tendance vers la dématérialisation des services financiers et particulièrement l'assurance qui fait l'objet de cet article qui se veut une analyse du cadre juridique marocain de la vente à distance dématérialisée (VAD) des services et particulièrement l'assurance.

\section{Méthodologie}

La méthodologie adoptée pour cette étude consiste à répondre à certaines questions posées au départ pour voir s'il y a un changement significatif au niveau du cadre juridique de présentation à distances des opérations d'assurances en premier lieu et de voir si ce cadre juridique réponds au attentes des opérateurs du secteur des assurances en matière de vente à distance dématérialisée de l'assurance (l'e-assurance). A cela s'ajoute une analyse critique sur les faiblesses de ce cadre juridique en faisant une comparaison entre la réglementation européenne en matière de vente à distance des services financier en lignes.

Pour ce faire, cet article s'appuie sur quatre points à savoir:

I. Le cadre juridique de la VAD des services financiers selon la loi 3108 ;

II. La circulaire $\mathrm{n}^{\circ} \mathrm{DAPS} / \mathrm{EA} / 12 / 19$ base réglementaire de la VAD dématérialisée de l'assurance au Maroc;

III. Le champ et les limites de la présentation des opérations d'assurances à distance; 
IV. La critique du cadre juridique de présentation de l'e-assurance;

La question qui se pose à première vue, existe-t-il, au Maroc, un cadre juridique propre de la vente à distance dématérialisée des services financiers en ligne ? c'est ce qui a été développé au niveau du premier point qui suit :

\section{Le cadre juridique de la VAD des services financiers selon la loi 31-08}

Contrairement à certaines législations, l'Union Européenne et au Royaume- Uni qui sont dotés de lois régissant le commerce électronique s'appliquant spécialement aux produits financiers (Comité du commerce électronique du conseil canadien des responsables de la réglementation d'assurance, 2012), le Maroc n'est pas doté d'un cadre juridique spécifique à la VAD qui s'applique expressément aux produits financiers.

Le Maroc est doté d'un cadre juridique composé de plusieurs lois, arrêtés, décrets et circulaires qui encadrent la vente des produits financiers, notamment les produits d'assurance qui fait l'objet de cet article.

La vente à distances des produits financiers, dont l'assurance fait partie, trouve sa base juridique dans l'art 26 al 1 de la loi 31-08, qui permet l'exercice de prestations de services à distance. Cela, comme le montre cet article, est la conséquence directe de l'absence d'un code monétaire et financier : grand obstacle pour la vente à distance des produits financiers !

L'absence d'un cadre, document ou code, englobant l'ensemble des aspects de la finance au Maroc est un handicap. La multitude de textes juridiques qui touchent aux services financiers et l'inexistence d'un recueil juridique qui regroupe ces différents textes, est un frein à la recherche, à la réforme et au développement des instruments financiers ; le projet de code monétaire et financier toujours en instance n'a pas encore vu le jours (Rahal, 2018).

La loi 31-08 relative à la protection du consommateur a permis la vente à distance des services financiers. Toutefois, cette possibilité de vendre à distance des services financiers manque d'un cadre juridique qu'il lui est propre. En effet, l'art 26 al 1 de ladite loi, se contente d'indiquer cette permission.

En effet, l'art 26 al 1 susmentionné est la base légale sur laquelle on s'appui pour vendre à distance les services financiers en ligne; il ne fait allusion qu'à la possibilité de vendre à distance les services de manière globale. Mais comme le montre cet article, un dispositif juridique aurait mieux valu pour encadrer la vente à distance des produits financiers ; d'ailleurs en droit comparé, français et européen qui sont une source d'inspiration et de benchmark, ont prévu un cadre juridique pour la vente à distance des produits financiers, on trouve en droit comparé la directive européenne 2002/65/CE 
relative à la vente à distance des services financiers qui est un cadre juridique qui cerne tous les aspects, particuliers, des services financiers.

De plus, contrairement au législateur français ou communautaire qui ont distingué entre les différents services vendus à distances, les services financiers à part et les services non financiers, le législateur marocain les a considéré sans distinction, en droit marocain la loi 31-08 parle de service de manière générale, ce qui a notre humble avis, vu l'importance des services financiers mériterait d'avoir un cadre juridique propre, qui s'impose puisqu'on constate la croissance exponentielle à moyen et long terme de la vente des services financiers en nombre et en masse monétaire (Rahal, 2018). En effet, une note de l'autorité de controle des assurances et de la prévoyance sociale (ACAPS) indique qu'en 2019, le chiffre d'affaires des entreprises d'assurances et de réassurance atteint 46,7 milliards de dirhams, en progression de $8,4 \%$ par rapport à $2018^{14}$.

Rangs du secteur marocain en termes de densité (2016-2018)

Source : Swiss Ré

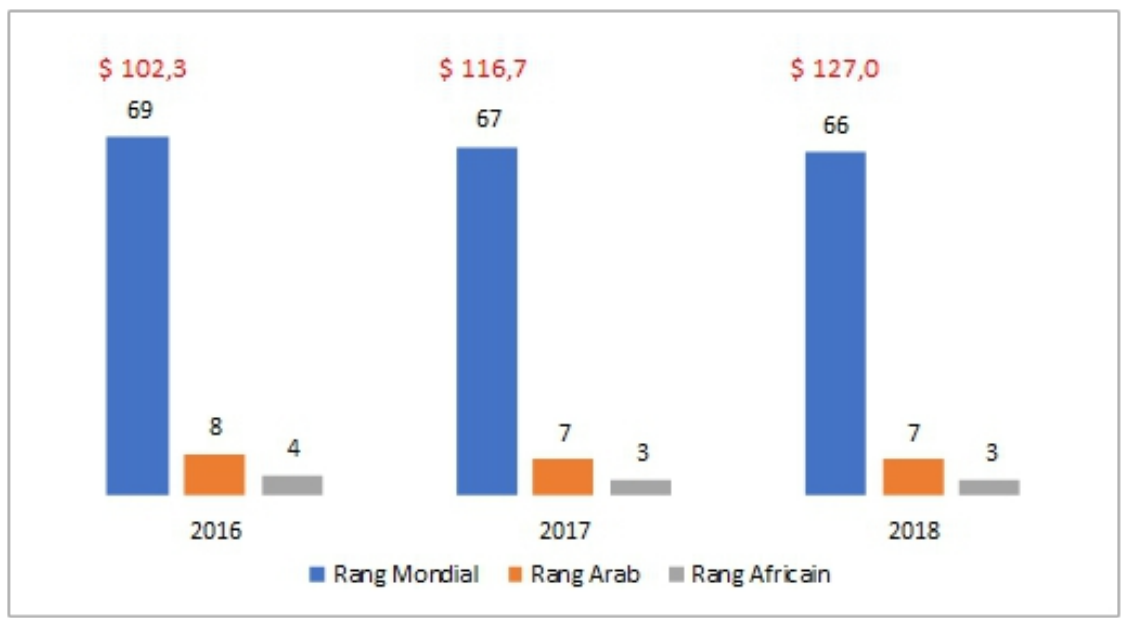

${ }^{14}$ Secteur des assurances et de la réassurance, situation 
Rangs du secteur marocain en termes de chiffres d'affaires (2016 - 2018)

Source : Swiss Ré

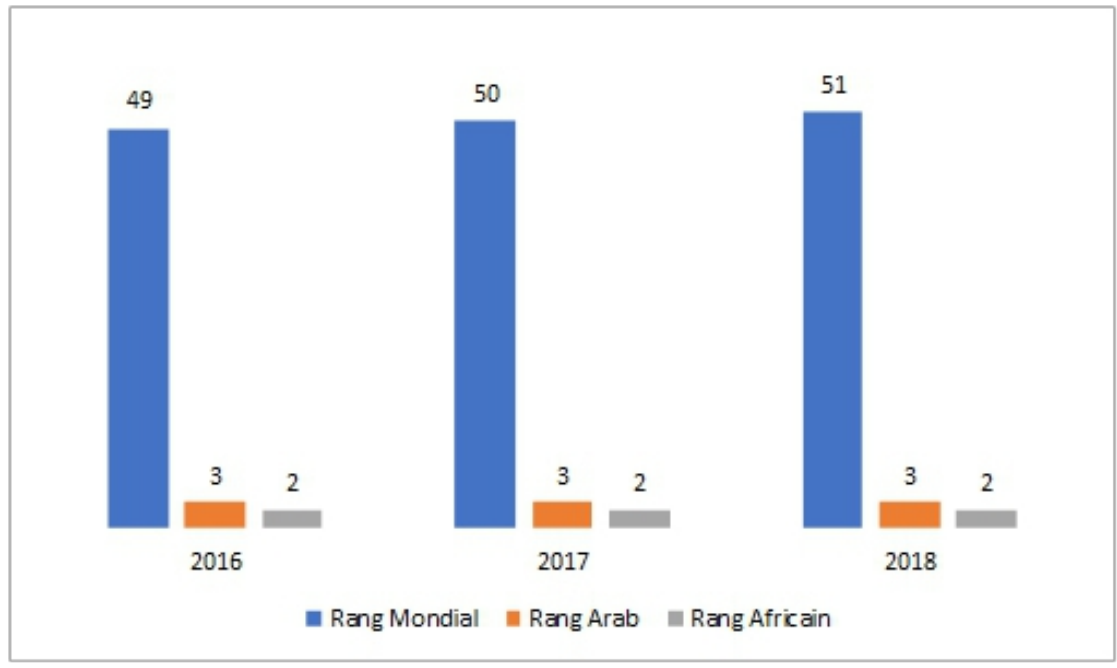

Trouve-t-on une définition de services financiers en ligne en droit marocain?

D'après les recherches que nous avons entamées, non, il n'existe aucune définition donnée par le droit marocain (Rahal, 2018) ceci nous amène à rechercher cette définition en droit comparé ou en doctrine. En effet, le terme de service financier a fait l'objet d'une définition précise, en droit communautaire Européen, dans la directive 2002/65/CE ; «Tout service ayant trait à la banque, au crédit, à l'assurance, aux retraites individuelles, aux investissements et aux paiements» (Directive 2002/65/CE du Parlement européen et du Conseil, 23 septembre 2002).

Après avoir présenté le cadre juridique globale de la vente à distance des services de manière générale on s'attachera, dans le point ci-après, à voir le cadre réglementaire de la VAD dématérialisée des services financiers de l'assurance au Maroc.

\section{La circulaire $n^{\circ}$ DAPS/EA/12/19 base réglementaire de la VAD dématérialisée de l'assurance au Maroc}

Le cadre juridique des services financiers en ligne est en cours de construction, d'ailleurs à travers cet article, on peut dire qu'il n'existe pas un cadre général unique, qui encadre la commercialisation et la vente à distance des services financiers au Maroc et particulièrement l'assurance qui nous intéresse (Rahal, 2018).

Mais loin de là, il existe un dispositif en cours de construction. Ce dispositif s'appuie sur la loi 31-08 relative à la protection du consommateur qui a consacré tout le chapitre 2 , les contrats conclus à distance, de l'article 25 à l'article 44. 
Toutefois, c'est la circulaire ${ }^{\circ}$ DAPS/EA/12/19 du 09 mars 2012 relative à la fourniture à distance d'opérations d'assurances, qui est venu encadrer la vente à distance des produits d'assurances au Maroc.

Ce n'est que par la suite, qu'un projet de loi a été introduit dans le circuit, toujours, en instance, au Secrétariat général du gouvernement (SGG), se proposant de modifier et compléter la loi 17-99 portant code des assurances. Ce projet de loi vise à introduire le dispositif de vente à distance des produits d'assurance dans le cadre juridique, qu'est la loi 17-99. Est ce que c'est la bonne approche?, réglementer à traver une circulaire uniquement sur les produits d'assurance. Ne valait-il pas mieux instaurer un cadre juridique globale pour les services financiers.

La loi bancaire, qui encadre la vente des produits financiers bancaire, n'a rien prévu aussi en matière de vente à distance de ces services financier en ligne, absence de dispositions au niveau de la loi bancaire $\mathrm{n}^{\circ}$ 103.12. Les banques sont, également des distributeurs de produits d'assurances, y compris l'e-assurances. A cela s'ajoute l'aspect des paiements à distances des services financiers, on ne trouve pas un encadrement juridique, assez développé, qui se focalise sur cet aspect.

Après avoir présenté le cadre juridique de la VAD de l'assurance on va se focaliser sur le champ et les limites de cette présentation.

\section{Le Champ et les limites de la présentation des opérations d'assurances à distance}

Dans cet article, il a été question de voir (i) l'étendue de la présentation des opérations d'assurance à distance, et de faire ressortir (ii) des limites juridiques à la VAD dématérialisée des produits d'assurances.

i. Etendue de la présentation des opérations d'assurance à distance

La note introductive de la circulaire n ${ }^{\circ}$ DAPS/EA/12/19 du 09 mars 2012 relative à la fourniture à distance d'opérations d'assurances, a cerné les personnes ou les opérateurs qui peuvent vendre à distance les produits d'assurance, il s'agit des entreprises d'assurances et de réassurance, des intermédiaires d'assurances et toutes autres entités autorisées à présenter les opérations d'assurances souhaitant exploiter les différents moyens mis à leur disposition par les techniques de communication pour la fourniture à distance d'opérations d'assurances.

A première vu aucun nouveau intervenant dans le champ de la distribution des produits d'assurance. Donc les distributeurs classiques que sont les entreprises d'assurances et de réassurance via les bureaux directs, les intermédiaires d'assurances (agents et courtiers d'assurances) et les nouveaux intervenants dans le champ de la distribution (les Banques, les Sociétés de 
financements, AL BARID BANQUE (ex-Barid al Maghreb) et les sociétés de micro-crédit.

Cela montre que le pouvoir administratif via l'article 306 al 5 de la loi $\mathrm{n}^{\circ}$ 17-99 portant code des assurances qui a permis l'introduction des sociétés de financement et $\mathrm{Al}$ Barid bank, permet toujours d'introduire à tout moment un nouvel acteur dans le champ de la distribution par simple arrêté du Ministère de l'Economie et des Finances, après accords de l'autorité de contrôle des assurances et de la prévoyance sociale (ACAPS).

Donc rien n'empêche qu'une nouvelle entité autorisée à présenter des produits d'assurances ne le fasse de manière totalement dématérialisée. Ceci est d'autant plus conforté par l'art 306 susmentionné qui permet au pouvoir exécutif d'introduire de nouveaux acteurs dans le champ de la distribution sans passer par le circuit législatif.

Toutefois, l'étendue de la présentation des opérations d'assurances à distance de manière dématérialisée n'est pas sans limite.

ii. Les limites juridiques à la VAD dématérialisée des produits d'assurances

Les exigences juridiques et administratives préalable à la VAD des produits d'assurances sont à respecter.

En effet, tous les produits d'assurances ne peuvent être vendus à distance par les opérateurs, que sous certaines conditions juridiques. En effet, ne peuvent être fournis à distance que les contrats d'assurances, dont les spécimens ont été transmis à l'ACAPS en application de l'article 247 de la loi $\mathrm{n}^{\circ} 17-99^{15}$, ayant fait l'objet d'une décision relative à leur commercialisation ou n'ayant pas soulevé d'observations de la part de l'ACAPS dans le délai de 30 jours.

Ceci montre que la VAD des produits d'assurances en ligne, subit le contrôle de l'entité chargée de l'exercer à savoir l'ACAPS. Vendre à distance les produits d'assurance, outre, l'autorisation stipulée, les conditions mentionnées et les préalables demandés conformément à la loi 17-99 portant code des assurances ou des textes pris pour son application, exige la transmission à l'ACAPS des éléments suivants :

\footnotetext{
${ }^{15}$ Art 247 dispose :

«Les spécimens des contrats d'assurance que les entreprises d'assurances et de réassurance entendent émettre pour la première fois doivent être communiqués à l'administration préalablement à leur émission. Outre les spécimens de contrats d'assurance, l'administration peut exiger la communication de tous documents à caractère contractuel ou publicitaire ayant pour objet une opération d'assurance ou de réassurance.

S'il apparaît qu'un document est contraire aux dispositions de la présente loi ou des textes pris pour son application, l'administration peut en exiger la modification ou en décider le retrait. En l'absence d'observation de la part de l'administration dans un délai de trente (30) jours à compter de leur réception, les documents peuvent être distribués, remis ou diffusés ».
} 
- Les spécimens des contrats d'assurance que les entreprises d'assurances et de réassurance entendent émettre pour la première fois ;

- Éventuellement, la communication de tous documents à caractère contractuel ou publicitaire ayant pour objet une opération d'assurance ou de réassurance.

Les éléments transmis à l'ACAPS font l'objet d'un contrôle, et s'il apparaît qu'un document est contraire aux dispositions de la loi 17-99 portant code des assurances ou des textes pris pour son application, l'ACAPS peut en exiger la modification ou en décider le retrait.

Passé le délai de 30 jours, et n'ayant aucune réponse de l'ACAPS, l'opérateur pourra vendre le produit en question.

\section{Analyse critique du cadre juridique de présentation de l'e- assurance}

A travers cet article, on a mis en exergue les conditions juridiques de présentation des opérations d'assurance en ligne (i), on a démontré l'insuffisance du cadre juridique de vendre à distance de l'assurance qui est en cours de construction (ii) et émis des propositions d'amélioration.

\section{i. $\quad$ Conditions juridiques de présenter l'assurance en ligne}

Le cadre juridique de l'e-assurance est permis par la loi 31-08 relative à la protection du consommateur qui a donné la possibilité de vendre à distance les services. ${ }^{16}$

Ce n'est qu'à la suite de ce vide juridique laissé par le législateur, que le pouvoir exécutif a édicté la circulaire n ${ }^{\circ}$ DAPS/EA/12/19 du 09 mars 2012 relative à la fourniture à distance d'opérations d'assurances, pour parer à la pression que les assureurs ont pesé, de vendre à distance l'assurance puisque aucune disposition juridique ne l'interdit et aucun cadre réglementaire ne le prévoyait, ni encadrait le déroulement et le cheminement de cette vente à distance dématérialisée.

Pour pouvoir exercer la VAD de l'assurance il faut des conditions juridiques à respecter :

- Ettre un opérateur agréé par l'ACAPS, pour pouvoir vendre à distance les produits d'assurances ${ }^{17}$; il ne suffit pas d'avoir l'agrément pour distribuer les produits d'assurances, mais il faut en plus une autre autorisation qui permet de vendre à distance, sollicité auprès de l'autorité de contrôle ;

\footnotetext{
${ }^{16}$ Art 30 de loi 31-08 édictant des mesures de protection du consommateur.

${ }^{17}$ Art 3 de la circulaire ${ }^{\circ}$ DAPS/EA/12/19 du 09 mars 2012 relative à la fourniture à distance d'opérations d'assurances.
} 
- Avoir un local autorisé par l'ACAPS et dirigé par un salarié ayant les compétences techniques requises en matière de présentation des opérations d'assurances sous la responsabilité du représentant responsable ou du salarié responsable de l'opérateur ${ }^{18}$.

Pour le cas d'extension, de prestation de services, d'un agrément déjà existant, à notre humble avis, il ne suffit pas d'avoir l'ancien agrément, il faudra prévoir une extension de l'agrément et de l'autorisation pour pouvoir vendre à distance de manière dématérialisée les nouveaux services ; d'ailleurs on trouve en droit comparé, la doctrine française, Mathieu M-E, pour le cas d'une extension de l'agrément ${ }^{19}$ et une information préalable aux services d'accueil seront nécessaires avant toute offre de nouveaux services non agréés en ligne (Boulaich Bayssa, 2012).

Selon la réglementation marocaine le fait d'être agréé pour vendre certains services de l'assurances ne suffit pas, il faut en outre une autorisation spéciale pour vendre à distance de manière dématérialisée tel que stipulé par la circulaire de l'ACAPS.

L'encadrement juridique relatif à la VAD des produits d'assurances parle de compétences techniques du salarié qui sera chargé de cet aspect sans expliciter les compétences techniques requises, ni les exigences que doit avoir ce salarié.

L'exigence d'un local autorisée par l'ACAPS, est à notre sens une manière d'éviter d'avoir un opérateur de présentation exerçant la VAD de l'assurance de manière dématérialisée à $100 \%$. Ce qui est à notre sens un frein au tout digitale et à la dématérialisation totale des transactions financière en ligne. L'ouverture de l'assurance à l'internet a donc soulevée un problème juridique de localisation du lieu où le service dématérialisé sera effectué, cette exigence est prévue probablement afin d'éviter d'avoir un opérateur difficilement localisable exerçant son activité sur la toile. C'est un argument qui intenable, puisque pour pouvoir vendre l'assurance un agrément est nécessaire; de ce fait, la personne qui veut présenter les opérations d'assurances est identifiable.

Mais cette exigence d'un locale physique ne risque-t-elle pas de freiner la migration de l'assurance vers la dématérialisation et delà handicaper la préparation des assureurs aux mutations, qui se profilent, dû à la digitalisation future de l'assurance.

${ }^{18}$ Ibid.

${ }^{19} \mathrm{Si}$ le prestataire souhaite exercer en ligne des services pour lesquels il n'a pas été agréé. 
ii. $\quad$ Un cadre juridique en cours de construction imparfait

Le cadre juridique des services financiers en ligne est en cours de construction, car à notre humble avis, et à travers cet article, on peut constater qu'il n'existe pas un cadre général unique, qui encadre la commercialisation et la vente à distance des services financiers au Maroc et particulièrement l'assurance qui nous intéresse.

Mais loin de là, il existe un dispositif en cours de construction. Ce dispositif s'appuie sur la loi 31-08 relative à la protection du consommateur qui a consacré tout le chapitre 2 , les contrats conclus à distance, de l'article 25 à l'article 44 .

Nous avons, aussi, la circulaire nDAPS/EA/12/19 du 09 mars 2012 relative à la fourniture à distance d'opérations d'assurances, qui est venu encadrer la vente à distance des produits d'assurances.

Ce n'est que par la suite, qu'un projet de loi a été mis dans le circuit du secrétariat général du gouvernement (SGG) proposant de modifier et compléter la loi 17-99 portant code des assurances, en instance toujours. Ce projet de loi vise à introduire le dispositif de vente à distance des produits d'assurance dans le cadre juridique qu'est la loi 17-99 en modifiant le livre IV, titre IV les Articles 330-3 et 330-4.

Article 330-3 : dispose la présentation des opérations d'assurances au moyen d'une technique de communication à distance est régie par les dispositions de la présente loi, de la loi n³1-08 édictant des mesures de protection du consommateur, notamment le chapitre 2 du titre IV et de la loi n`53-05 relative à l'échange électronique des données juridiques.

Ladite présentation ne peut s'effectuer que par les personnes visées à l'article 290 de la loi 17-99 à l'exception des démarcheurs. Ces personnes doivent, au préalable, en informer l'Autorité (ACAPS).

Articles 330-4 : «la présentation des opérations d'assurances au moyen d'une technique de communication à distance ne peut être exercée qu'à partir d'un local au sens de l'article 307 de la loi 17-99 et par un salarié ayant les compétences techniques requises en matière de présentation des opérations d'assurances.

Les modalités de la présentation des opérations d'assurances au moyen d'une technique de communication à distance sont fixées par circulaire de l'Autorité ».

Les deux projets d'articles Article 330-3 et Articles 330-4, toujours en instances, ne peut apporter un éclairage dans le monde de la présentation à distance des opérations d'assurances. En effet, le marché de l'assurance se transforme, l'ACAPS se doit d'apporter des réponses concrètes et d'anticiper sur les mutations profondes qui se profilent. D'ailleurs les deux propositions d'articles viennent uniquement conforter les dispositions de la circulaire $\mathrm{n}^{\circ} \mathrm{DAPS} / \mathrm{EA} / 12 / 19$ et n'apportent pas un changement majeur. 
On pourrait aussi ajouter, dans un autre registre, la loi bancaire, qui encadre la vente des produits financiers bancaire, n'a rien prévu aussi en matière de vente à distance de ces services financier en ligne, absence de dispositions au niveau de la loi bancaire $\mathrm{n}^{\circ} 103.12$ Les banques sont, également des distributeurs de produits d'assurances, y compris l'eassurances. A cela s'ajoute l'aspect des paiements à distances des services financiers, on ne trouve aucun encadrement juridique qui se focalise, avec pertinence, sur cet aspect.

\section{iii. Propositions d'amélioration}

Cet analyse critique révèlle des insuffisances à palier, à savoir :

- En premier lieu prévoir un cadre juridique spécifique à la vente à distance dématérialisée des services financiers à travers une directive commune entre $\mathrm{BAM}^{20}$, l'ACAPS, l'AMMF$^{21}$ et le $\mathrm{MEF}^{22}$.

- En deuxième lieu consacrer aux instruments de paiement en ligne un cadre qui clarifie son mode d'utilisation pour avoir une visiblité sur tous le processus lors de la vente à distance dématérialisé des services financiers. La loi bancaire doit étre revue au niveau des instruments de paiement en ligne.

- La loi 31-08 relative à la protection du consommateur doit être revue dans le sens de proteger davantage l'e-consommateur à travers des régles qui imposent aux assureurs une information effective sur ses droits et obligations.

- Les données présentées dans le rapport d'activité de 1'ACAPS, ne permettent pas de distinguer le nombre de contrat conclus à distance de manière dématérialisée. Prévoir au sein du rapport des élèments qui font ressortir le moyen avec lequel le contrat d'assurance a été conclu.

\section{Conclusion}

Le cadre juridique de l'assurance est en pleine mutation, la manière de présenter les opérations d'assurances au Maroc se transforme doucement à travers la venue de nouveaux acteurs dans le champ de la distribution. La VAD dématérialisée de l'assurance à travers internet risque de modifier totalement le paysage de présentation des opérations d'assurance.

Il est vrai que le service financier issus de l'assurances est technique et demande un suivi et un contact direct des consommateurs, non initiés, auprès des opérateurs de l'assurance. Mais cela, ne peut être un frein au développement du tout digital ou de combiner à la fois le digital et le contact

\footnotetext{
${ }^{20}$ Bank Al-Maghrib

${ }^{21}$ L'autorité marocaine des marchés financiers

${ }^{22}$ Ministère de l'économie et des finances
} 
direct avec les assureurs. Pour certains produits la digitalisation peut se faire, pour d'autres cela nécessitera plus de temps et de maturité. Les parts de marché se jouent maintenant. Toutefois, sur le terrain la dématérialisation total du contrat d'assurance tarde à percer, en atteste, au Maroc, la transmission par courrier classique du contrat d'assurance papier de la part de l'assureur à l'assuré à travers les services postaux, transmis par des sociétés de services de transport de colis tel que AMANA (société de transmission de colis).

On peut avancer à la lumière des développements présentés au niveau de cet article plusieurs insuffisances du cadre juridique au Maroc qui encadre la vente dématérialisée de l'assurance, à savoir:

- l'absence d'un cadre englobant l'ensemble des aspects de la finance au Maroc est un handicap est un frein à la recherche, à la réforme et au développement des instruments financiers. On peut dire qu'au Maroc vendre à distance les services financiers manque d'un cadre juridique qu'il lui est propre.

- l'inexistence d'un cadre général unique, qui encadre la commercialisation et la vente à distance des services financiers au Maroc et particulièrement l'assurance fait défaut; d'ailleurs la mise en place d'un dispositif juridique spécifique aurait mieux valu pour encadrer la vente à distance des produits financiers. Une directive générale de la part des différents acteurs (BAM, ACAPS, AMMF et le $\mathrm{MEF}$ ) est souhaitable.

- le cadre juridique actuel (code des assurances) ne permet pas de dématérialiser totalement le processus de vente à distance puisque les opérateurs d'assurances dans la phase ultime du contrat d'assurances utilisent toujours le contrat papier. La dématérialisation est imparfaite.

L'ACAPS ne peut retarder le changement, ni le freiner à travers l'instauration de règles qui limitent la souscription en ligne de contrat d'assurance, au risque de voir les opérateurs marocains du secteur des assurances en retard par rapport à leurs concurrents sur le marché africain. Il est souhaitable que l'ACAPS produisent un état au niveau de son rapport d'activité des statistiques sur la vente en ligne de l'assurance.

Le cadre juridique de la VAD des services financiers gagnerait à être encadré par un dispositif juridique à même de lui apporter un socle qui puisse regrouper, consigner et guider dans l'accès aux dispositions qui existe déjà et pallier aux carences qu'on rencontre au niveau du cadre juridique actuel.

Finalement, dans ces moments de crise dû au covid 19, qui a pour beaucoup freiner la dynamique économique du monde et non pas seulement du pays, nous enseigne que le télétravail, l'utilisation des moyens techniques à distance, l'internet, ...etc, sont salutaires dans beaucoup de situations. Ceci est valable pour l'e-assurance qui dans un contexte pareille est amenée à 
amortir les risques des acteurs économiques. La VAD dématérialisée de l'assurance en ligne s'impose et le législateur marocain, les assureurs et les pouvoir publics doivent s'inscrire dans cette dynamique.

\section{References:}

1. Boulaich Bayssa, F-Z. (2012). Les prestations financières en ligne, Thèse pour le Doctorat en Droit, Université Paris I (PanthéonSorbonne), France, 423 Pages.

2. Chahafi, Y. (2020). L'instauration d'une approche basée sur les risques dans la lutte contre le blanchiment des capitaux et le financement du terrorisme dans le secteur des assurances, PFE, univesrsité IBN TOFAIL, Kénitra, Maroc, 87 Pages.

3. Circulaire ${ }^{\circ} \mathrm{DAPS} / \mathrm{EA} / 12 / 19$ du 09 mars 2012 relative à la fourniture à distance d'opérations d'assurances.

4. Circulaire du président de l'autorité de contrôle des assurances et de la prévoyance sociale $\mathrm{n}^{\circ} 01 / \mathrm{AS} / 19$ du 2 janvier 2019 prise pour l'application de certaines dispositions de la loi 17-99 portant code des assurances, BO n 6778 - 10ramadan 1440 (16-5-2019).

5. Directive 2002/65/CE du Parlement européen et du Conseil du 23 septembre 2002 concernant la commercialisation à distance de services financiers, Journal officiel $\mathrm{n}^{\circ} \mathrm{L} 271 \mathrm{du}$ 09/10/2002 p. 0016 - 0024.

6. Le comité du commerce électronique du conseil canadien des responsables de la réglementation d'assurance. (2012). Le commerce Electronique des produits d'assurance.

Source :

http://www.ccirccrra.org/fr/init/Elec_Commerce/ECC\%20issues\%20paper\%20\%20F R2.pdf

7. Loi n'59-13 modifiant et complètent la loi 17-99 portant code des assurances. BO n65-06 du 6 octobre 2016.

8. Loi $\mathrm{n}^{\circ} 103-12$, relative aux établissements de crédits et organismes assimilés, B.O nº 634014 joumada I 1436 (5 mars 2015).

9. Loi $\mathrm{n}^{\circ} 31-08$ édictant des mesures de protection du consommateur promulguée par le dahir $\mathrm{n}^{\circ} 1-11-03$ du 14 rabii I, 1432. B.O n $5932 \mathrm{du}$ 7 avril 2011.

10. Loi 17-99 portant code des assurances promulguée par le dahir $n^{\circ} 1$ 02-238 du 3 octobre 2002. BO n5054 du 7 novembre 2002.

11. Rahal, H. (2018). La vente à distance dématérialisée par internet : cas du contrat d'assurance, Thèse de doctorat, spécialité droit des assurances, Université Abdelmalak saidi, Tanger, Maroc, 460 pages.

12. Rahal, H. (2014). La distribution des produits d'assurances au Maroc tendances et défis, Edition Dar al Qalam, Rabat, 209 pages. 